DFTT 32/99

hep-ph/9906275

\title{
Neutrinoless double- $\beta$ decay with three or four neutrino mixing
}

\author{
Carlo Giunti \\ INFN, Sezione di Torino, and Dipartimento di Fisica Teorica, Università di Torino, \\ Via P. Giuria 1, I-10125 Torino, Italy
}

\begin{abstract}
Considering the scheme with mixing of three neutrinos and a mass hierarchy that can accommodate the results of solar and atmospheric neutrino experiments, it is shown that the results of solar neutrino experiments imply a lower bound for the effective Majorana mass in neutrinoless double- $\beta$ decay, under the natural assumptions that massive neutrinos are Majorana particles and there are no unlikely fine-tuned cancellations among the contributions of the different neutrino masses. Considering the four-neutrino schemes that can accommodate also the results of the LSND experiment, it is shown that only one of them is compatible with the results of neutrinoless double- $\beta$ decay experiments and with the measurement of the abundances of primordial elements produced in Big-Bang Nucleosynthesis. It is shown that in this scheme, under the assumptions that massive neutrinos are Majorana particles and there are no cancellations among the contributions of the different neutrino masses, the results of the LSND experiment imply a lower bound for the effective Majorana mass in neutrinoless double- $\beta$ decay.
\end{abstract}

PACS numbers: 23.40.-s, 14.60.Pq, 14.60.St 


\section{INTRODUCTION}

Neutrino oscillations [1] is one of the most intriguing phenomena of today high-energy physics and one of the most promising ways to explore the physics beyond the Standard Model. At present there are three experimental indications in favor of neutrino oscillations that have been obtained in solar neutrino experiments (Homestake [4], Kamiokande [5], GALLEX [6], SAGE [7] and Super-Kamiokande [8]), in atmospheric neutrino experiments (Kamiokande [9], IMB [10], Super-Kamiokande [11], Soudan-2 [12] and MACRO [13])) and in the accelerator LSND experiment [14,15]. On the other hand, neutrinoless double- $\beta$ decay $\left(\beta \beta_{0 \nu}\right)$ experiments [16 19] and the experiments on the direct measurement of neutrino masses [20] did not obtain any positive result so far (see [21]). The connection between the properties of neutrinos that determine neutrino oscillations (mass squared differences and neutrino mixing) and neutrinoless double- $\beta$ decay have been discussed in many papers 22233 . In this paper we discuss some implications of the latest results of neutrino oscillation experiments for neutrinoless double- $\beta$ decay and we show that under reasonable assumptions there is a lower bound for the effective Majorana neutrino mass measured in $\beta \beta_{0 \nu}$ decay experiments.

If massive neutrinos are Majorana particles, the matrix element of $\beta \beta_{0 \nu}$ decay is proportional to the effective Majorana mass

$$
|\langle m\rangle|=\left|\sum_{k} U_{e k}^{2} m_{k}\right|,
$$

where $U$ is the mixing matrix that connects the flavor neutrino fields $\nu_{\alpha L}(\alpha=e, \mu, \tau)$ to the fields $\nu_{k L}$ of neutrinos with masses $m_{k}$ through the relation

$$
\nu_{\alpha L}=\sum_{k} U_{\alpha k} \nu_{k L}
$$

The present experimental upper limit for $|\langle m\rangle|$,

$$
|\langle m\rangle|_{\exp } \leq 0.2-0.4 \mathrm{eV} \quad(90 \% \mathrm{CL}),
$$

has been obtained from the measurement of the half-life of ${ }^{76} \mathrm{Ge}$ in the Heidelberg-Moscow experiment $\left(T_{1 / 2}^{0 \nu}\left({ }^{76} \mathrm{Ge}\right) \geq 5.7 \times 10^{25} \mathrm{yr}\right.$ at $\left.90 \% \mathrm{CL}\right)$ [18. The range of the upper bound (1.3) is due to the uncertainty of the theoretical calculation of the nuclear matrix element and has been obtained from the results of different calculations using the Quasiparticle Random Phase Approximation (QRPA) [34,35]. In particular, the recent QRPA calculation in 35 yields the rather stringent upper bound $|\langle m\rangle|_{\exp } \leq 0.27 \mathrm{eV}$. On the other hand, the Shell Model calculation in [36] yields the loser bound $|\langle m\rangle|_{\exp } \leq 0.56 \mathrm{eV}$. However, the calculation of the nuclear matrix element for the neutrinoless double- $\beta$ decay of ${ }^{76} \mathrm{Ge}$ presented in [36] has been truncated before reaching convergence and the full calculation is expected to yield

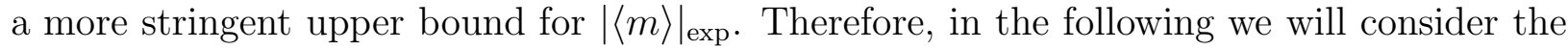
range in Eq. (1.3) as a reliable estimate of the uncertainty of the experimental upper bound for the effective Majorana mass $|\langle m\rangle|$. 
The next generation of $\beta \beta_{0 \nu}$ decay experiments is expected to be sensitive to values of $|\langle m\rangle|$ in the range $10^{-2}-10^{-1} \mathrm{eV}$ [17]. Values of $|\langle m\rangle|$ as small as about $10^{-3} \mathrm{eV}$ may be reachable not far in the future [19].

After the measurement in the Super-Kamiokande experiment of an up-down asymmetry of $\mu$-like events induced by atmospheric muon neutrinos, the experimental evidence in favor of oscillations of atmospheric neutrinos is widely considered to be beyond reasonable doubts (see, for example, [2,37,3]). There are also convincing arguments in favor of a neutrino oscillation explanation of the solar neutrino problem (see, for example, 38,2, 2,39]). Therefore, in this paper we will consider first, in Section $\mathbb{1}$, the implications for $\beta \beta_{0 \nu}$ decay in the scheme with mixing of three neutrinos and a mass hierarchy that can accommodate the results of atmospheric and solar neutrino experiments. In Section III we consider the schemes with four massive neutrinos that can accommodate all neutrino oscillation data, including the LSND results in favor of $\nu_{\mu} \rightarrow \nu_{e}$ and $\bar{\nu}_{\mu} \rightarrow \bar{\nu}_{e}$ oscillations that wait for independent confirmations by other experiments 40,41].

\section{THREE NEUTRINOS WITH A MASS HIERARCHY}

The results of solar and atmospheric neutrino experiments indicate the existence of a hierarchy of mass-squared differences (see [38,42 45, 11]):

$$
\Delta m_{\mathrm{sun}}^{2} \lesssim 10^{-4} \mathrm{eV}^{2} \ll 10^{-3} \mathrm{eV}^{2} \lesssim \Delta m_{\mathrm{atm}}^{2} \lesssim 10^{-2} \mathrm{eV}^{2}
$$

where $\Delta m_{\text {sun }}^{2}$ and $\Delta m_{\text {atm }}^{2}$ are the mass-squared differences relevant for solar and atmospheric neutrino oscillations, respectively. A natural scheme that can accommodate this hierarchy is the one with three neutrinos and a mass hierarchy,

$$
\underbrace{\overbrace{m_{1} \ll m_{2}}^{\text {sun }} \ll m_{3}}_{\text {atm }} .
$$

In the framework of the hierarchical spectrum (2.2) the mass-squared differences relevant for the oscillations of solar and atmospheric neutrinos are

$$
\Delta m_{\mathrm{sun}}^{2}=\Delta m_{21}^{2} \equiv m_{2}^{2}-m_{1}^{2} \simeq m_{2}^{2}, \quad \Delta m_{\mathrm{atm}}^{2}=\Delta m_{31}^{2} \equiv m_{3}^{2}-m_{1}^{2} \simeq m_{3}^{2}
$$

The mass hierarchy (2.2) is predicted by the see-saw mechanism [46], which predicts also that the tree light massive neutrinos are Majorana particles. In this case neutrinoless double- $\beta$ decay is possible.

It has been shown in 25]27 that the results of neutrino oscillation experiments imply a rather stringent upper bound (about $6 \times 10^{-3} \mathrm{eV}$ ) for the effective Majorana mass in neutrinoless double- $\beta$ decay in the scheme with mixing of three neutrinos and a mass hierarchy. In principle the effective Majorana mass (1.1) can be vanishingly small because of cancellations among the contributions of the different mass eigenstates. However, since the neutrino masses and the elements of the neutrino mixing matrix are independent quantities, if there is a hierarchy of neutrino masses such a cancellation would be the result of an unlikely finetuning, unless some unknown symmetry is at work. Here we consider the possibility that no 
such symmetry exist and no unlikely fine-tuning operates to suppress the effective Majorana mass (1.1). In this case we have

$$
|\langle m\rangle| \simeq \max _{k}\left|U_{e k}\right|^{2} m_{k}
$$

Let us define the absolute value of the contribution of the neutrino mass $m_{k}$ to $|\langle m\rangle|$ as

$$
|\langle m\rangle|_{k} \equiv\left|U_{e k}\right|^{2} m_{k}
$$

In the following we will estimate the value of $|\langle m\rangle|$ using the largest $|\langle m\rangle|_{k}$ obtained from the results of neutrino oscillation experiments.

The results of the CHOOZ experiment 47 and the Super-Kamiokande atmospheric neutrino data [11] imply that $\left|U_{e 3}\right|^{2}$ is small $\left(\left|U_{e 3}\right|^{2} \lesssim 5 \times 10^{-2}\right)$ and there is an upper limit of about $6 \times 10^{-3} \mathrm{eV}$ for the contribution $|\langle m\rangle|_{3}$ to the effective Majorana mass in $\beta \beta_{0 \nu}$ decay 25 27]. Since there is no lower bound for $\left|U_{e 3}\right|^{2}$ from experimental data, $|\langle m\rangle|_{3}$ could be very small.

Hence, the largest contribution to $|\langle m\rangle|$ could come from $|\langle m\rangle|_{2} \equiv\left|U_{e 2}\right|^{2} m_{2}$. Since in the framework of the scheme with mixing of three neutrinos and a mass hierarchy $\Delta m_{\mathrm{sun}}^{2} \simeq m_{2}^{2}$ and $\left|U_{e 2}\right|^{2} \simeq \frac{1}{2}\left(1-\sqrt{1-\sin ^{2} 2 \vartheta_{\text {sun }}}\right)$ [48, where $\vartheta_{\text {sun }}$ is the two-neutrino mixing angle used in the analysis of solar neutrino data, we have

$$
|\langle m\rangle|_{2} \simeq \frac{1}{2}\left(1-\sqrt{1-\sin ^{2} 2 \vartheta_{\text {sun }}}\right) \sqrt{\Delta m_{\text {sun }}^{2}}
$$

Solar neutrino data imply bounds for $\sin ^{2} 2 \vartheta_{\text {sun }}$ and $\Delta m_{\text {sun }}^{2}$. In particular the large mixing angle MSW 49] solution (LMA-MSW) of the solar neutrino problem, which seems to be favored by recent data [50, implies that 42]

$$
1.2 \times 10^{-5} \mathrm{eV}^{2} \leq \Delta m_{\text {sun }}^{2} \leq 3.1 \times 10^{-4} \mathrm{eV}^{2}, \quad 0.58 \leq \sin ^{2} 2 \vartheta_{\text {sun }} \leq 1.00
$$

at $99 \% \mathrm{CL}$, taking into account the total rates measured in solar neutrino experiments and the day-night variations observed in the Super-Kamiokande experiment [8]. Hence, for the contribution of $m_{2}$ to the effective Majorana mass we obtain

$$
6 \times 10^{-4} \mathrm{eV} \lesssim|\langle m\rangle|_{2} \lesssim 9 \times 10^{-3} \mathrm{eV}
$$

This estimate does not take into account the correlation between $\Delta m_{\text {sun }}^{2}$ and $\sin ^{2} 2 \vartheta_{\text {sun }}$. The precise allowed range for $|\langle m\rangle|_{2}$ as a function of $\Delta m_{\text {sun }}^{2}$ obtained with Eq. (2.6) from the LMA-MSW region (99\% CL) in Fig. 2 of Ref. 442 is shown in Fig. 1. The dashed line in Fig. 1 represents the unitarity limit $|\langle m\rangle|_{2} \leq \sqrt{\Delta m_{\text {sun }}^{2}}$. From Fig. 11 one can see that the LMA-MSW solution of the solar neutrino problem implies that

1 The upper limit $|\langle m\rangle|_{2} \lesssim 3 \times 10^{-3} \mathrm{eV}$ presented in [27] has been obtained from the 90\% CL LMA-MSW region in Fig. 8a of Ref. 43], using Eq. (2.6). The 99\% CL LMA-MSW region in Fig. 8a of Ref. 43. gives $|\langle m\rangle|_{2} \lesssim 6 \times 10^{-3} \mathrm{eV}$, in agreement with the upper bound in Eq. (2.9). 


$$
7.4 \times 10^{-4} \mathrm{eV} \lesssim|\langle m\rangle|_{2} \lesssim 6.0 \times 10^{-3} \mathrm{eV} .
$$

Assuming the absence of fine-tuned cancellations among the contributions of the three neutrino masses to the effective Majorana mass, if $\left|U_{e 3}\right|^{2}$ is very small and $|\langle m\rangle|_{3} \ll|\langle m\rangle|_{2}$, from Eqs. (2.4) and (2.9) we obtain

$$
7 \times 10^{-4} \mathrm{eV} \lesssim|\langle m\rangle| \lesssim 6 \times 10^{-3} \mathrm{eV} .
$$

Hence, we see that, assuming the absence of an unlikely fine-tuned suppression of $|\langle m\rangle|$, the results of solar neutrino experiments give an indication of the value of the effective Majorana mass in $\beta \beta_{0 \nu}$ decay, with a lower bound of about $7 \times 10^{-4} \mathrm{eV}$ in the case of the LMA-MSW solution of the solar neutrino problem. This bound is rather small, but values of $|\langle m\rangle|$ of the order of $10^{-3} \mathrm{eV}$, indicated by the range (2.10), may be measurable in a not too far future [19.

Also the small mixing angle MSW (SMA-MSW) and the vacuum oscillation (VO) solutions of the solar neutrino problem imply allowed ranges for $|\langle m\rangle|_{2}$, but their values are much smaller than in the case of the LMA-MSW solution. Using the 99\% CL allowed regions obtained in [38] from the analysis of the total rates measured in solar neutrino experiments we have

$$
\begin{aligned}
& 5 \times 10^{-7} \mathrm{eV} \lesssim|\langle m\rangle|_{2} \lesssim 10^{-5} \mathrm{eV} \quad(\mathrm{SMA}-\mathrm{MSW}) \\
& 10^{-6} \mathrm{eV} \lesssim|\langle m\rangle|_{2} \lesssim 2 \times 10^{-5} \mathrm{eV} \quad(\mathrm{VO}) .
\end{aligned}
$$

\section{FOUR NEUTRINOS}

If, besides to the solar and atmospheric neutrino data, also the results of the accelerator LSND experiment are taken into account, at least three independent mass-squared differences are needed. This can be seen by considering the general expression for the probability of $\nu_{\alpha} \rightarrow \nu_{\beta}$ transitions in vacuum [1] 3], that can be written as

$$
P_{\nu_{\alpha} \rightarrow \nu_{\beta}}=\left|\sum_{k} U_{\alpha k}^{*} U_{\beta k} \exp \left(-i \frac{\Delta m_{k j}^{2} L}{2 E}\right)\right|^{2},
$$

where $\Delta m_{k j}^{2} \equiv m_{k}^{2}-m_{j}^{2}, j$ is any of the mass-eigenstate indices, $L$ is the distance between the neutrino source and detector and $E$ is the neutrino energy. The range of $L / E$ probed by each type of experiment is different: $L / E \gtrsim 10^{10} \mathrm{eV}^{-2}$ for solar neutrino experiments, $L / E \sim 10^{2}-10^{3} \mathrm{eV}^{-2}$ for atmospheric neutrino experiments and $L / E \sim 1 \mathrm{eV}^{-2}$ for the LSND experiment. From Eq. (3.1) it is clear that neutrino oscillations occur in an experiment only if there is at least one mass-squared difference $\Delta m_{k j}^{2}$ such that

$$
\frac{\Delta m_{k j}^{2} L}{2 E} \gtrsim 0.1
$$

(the precise lower bound depends on the sensitivity of the experiment) in a significant part of the energy and source-detector distance intervals of that experiment (if the condition (3.2) is 
not satisfied, $\left.P_{\nu_{\alpha} \rightarrow \nu_{\beta}} \simeq\left|\sum_{k} U_{\alpha k}^{*} U_{\beta k}\right|^{2}=\delta_{\alpha \beta}\right)$. Since the range of $L / E$ probed by the LSND experiment is the smaller one, a mass-squared difference is needed for LSND oscillations:

$$
\Delta m_{\mathrm{LSND}}^{2} \gtrsim 10^{-1} \mathrm{eV}^{2}
$$

Specifically, the maximum likelihood analysis of the LSND data in terms of two-neutrino oscillations gives [15]

$$
0.20 \mathrm{eV}^{2} \leq \Delta m_{\mathrm{LSND}}^{2} \leq 2.0 \mathrm{eV}^{2}
$$

Furthermore, from Eq. (3.1) it is clear that a dependence of the oscillation probability from the neutrino energy $E$ and the source-detector distance $L$ is observable only if there is at least one mass-squared difference $\Delta m_{k j}^{2}$ such that

$$
\frac{\Delta m_{k j}^{2} L}{2 E} \sim 1
$$

Since a variation of the transition probability as a function of neutrino energy has been observed both in solar and atmospheric neutrino experiments and the range of $L / E$ probed by each type of experiment is different, two more mass-squared differences with different scales are needed:

$$
\begin{aligned}
& \Delta m_{\mathrm{sun}}^{2} \sim 10^{-10} \mathrm{eV}^{2} \quad(\mathrm{VO}) \\
& \Delta m_{\mathrm{atm}}^{2} \sim 10^{-3}-10^{-2} \mathrm{eV}^{2} .
\end{aligned}
$$

The condition (3.6) for the solar mass-squared difference $\Delta m_{\text {sun }}^{2}$ has been obtained under the assumption of vacuum oscillations (VO). If the disappearance of solar $\nu_{e}$ 's is due to the MSW effect [49], the condition

$$
\Delta m_{\text {sun }}^{2} \lesssim 10^{-4} \mathrm{eV}^{2} \quad(\mathrm{MSW})
$$

must be fulfilled in order to have a resonance in the interior of the sun. Hence, in the MSW case $\Delta m_{\text {sun }}^{2}$ must be at least one order of magnitude smaller than $\Delta m_{\text {atm }}^{2}$.

The existence of three different scales of neutrino mass-squared differences: imply that at least four light massive neutrinos must exist in nature. Here we consider the schemes

${ }^{2}$ It is possible to ask if three different scales of neutrino mass-squared differences are needed even if the results of the Homestake solar neutrino experiment [4] is neglected, allowing an energyindependent suppression of the solar $\nu_{e}$ flux. The answer is that still the data cannot be fitted with only two neutrino mass-squared differences because an energy-independent suppression of the solar $\nu_{e}$ flux requires large $\nu_{e} \rightarrow \nu_{\mu}$ or $\nu_{e} \rightarrow \nu_{\tau}$ transitions generated by $\Delta m_{\mathrm{atm}}^{2}$ or $\Delta m_{\mathrm{LSND}}^{2}$. These transitions are forbidden by the results of the Bugey [51] and CHOOZ [47] $\bar{\nu}_{e}$ disappearance experiments and by the non-observation of an up-down asymmetry of $e$-like events in the SuperKamiokande atmospheric neutrino experiment [11]. I would like to thank S.T. Petcov for useful discussions about this point. 
with four light and mixed neutrinos [52 58], which constitute the minimal possibility that allows to explain all the data of neutrino oscillation experiments. In this case, in the flavor basis the three active neutrinos $\nu_{e}, \nu_{\mu}, \nu_{\tau}$ are accompanied by a sterile neutrino $\nu_{s}$ that does not take part in standard weak interactions.

The existence of four light massive neutrinos is a low-energy manifestation of physics beyond the Standard Model (see, for example, [59]). In most theories beyond the Standard Model neutrinos are naturally Majorana particles and neutrinoless double- $\beta$ decay is allowed. Therefore, we see that the experimental evidences in favor of neutrino oscillations indicate that neutrinos may be Majorana particles and neutrinoless double- $\beta$ decay is a concrete possibility.

It has been shown [55] that there are only two schemes with four-neutrino mixing that can accommodate the results of all neutrino oscillation experiments:

$$
\underbrace{\overbrace{m_{1}<m_{2}}^{\text {atm }}<\overbrace{m_{3}<m_{4}}^{\text {sun }}}_{\text {LSND }},
$$

$$
\underbrace{\overbrace{m_{1}<m_{2}}^{\text {sun }}<\overbrace{m_{3}<m_{4}}^{\text {atm }}}_{\text {LSND }} .
$$

These two spectra are characterized by the presence of two pairs of close masses separated by a gap of about $1 \mathrm{eV}$ which provides the mass-squared difference $\Delta m_{\mathrm{LSND}}^{2}=\Delta m_{41}^{2}$ responsible of the oscillations observed in the LSND experiment. In the scheme A $\Delta m_{\text {atm }}^{2}=\Delta m_{21}^{2}$ and $\Delta m_{\text {sun }}^{2}=\Delta m_{43}^{2}$, whereas in scheme $\mathrm{B} \Delta m_{\text {atm }}^{2}=\Delta m_{43}^{2}$ and $\Delta m_{\text {sun }}^{2}=\Delta m_{21}^{2}$.

The results of the short-baseline $\bar{\nu}_{e}$ disappearance experiment Bugey [51], in which no indication in favor of neutrino oscillations was found, imply that the mixing of $\nu_{e}$ with the two "heavy" neutrinos $\nu_{3}$ and $\nu_{4}$ is large in scheme A and small in scheme B [55,2]:

$$
\begin{aligned}
1-\left(\left|U_{e 3}\right|^{2}+\left|U_{e 4}\right|^{2}\right) & \lesssim 3 \times 10^{-2} \quad(\mathrm{~A}), \\
\left|U_{e 3}\right|^{2}+\left|U_{e 4}\right|^{2} & \lesssim 3 \times 10^{-2} \quad \text { (B) },
\end{aligned}
$$

for $\Delta m_{\mathrm{LSND}}^{2}$ in the LSND-allowed range (3.4). Therefore, if scheme $\mathrm{A}$ is realized in nature the effective Majorana mass in $\beta \beta_{0 \nu}$ decay can be as large as $m_{3} \simeq m_{4} \simeq \sqrt{\Delta m_{\mathrm{LSND}}^{2}} \simeq$ $0.45-1.4 \mathrm{eV}$ [24 27]. On the other hand, in scheme $\mathrm{B}$ neutrinoless double- $\beta$ decay is strongly suppressed [25 27]. In the following two subsections we discuss some connections between the results of neutrino oscillation experiments and neutrinoless double- $\beta$ decay in the schemes A and B.

\section{A. Scheme A}

In the four-neutrino scheme A, from Eq. (3.10) and the unitarity of the mixing matrix we have $\left|U_{e 1}\right|^{2}+\left|U_{e 2}\right|^{2} \lesssim 3 \times 10^{-2}$. Therefore, the contribution of the two light masses $m_{1}$ and $m_{2}$ to the effective Majorana mass in $\beta \beta_{0 \nu}$ decay can be neglected and we have

$$
|\langle m\rangle| \simeq\left|U_{e 3}^{2} m_{3}+U_{e 4}^{2} m_{4}\right|
$$

that implies the limits

$$
\left.|| U_{e 3}\right|^{2} m_{3}-\left.\left|U_{e 4}\right|^{2} m_{4}|\lesssim|\langle m\rangle|\lesssim| U_{e 3}\right|^{2} m_{3}+\left|U_{e 4}\right|^{2} m_{4}
$$


Neglecting the small difference between $m_{3}$ and $m_{4}$ and taking into account that $m_{3} \simeq m_{4} \simeq$ $\sqrt{\Delta m_{\mathrm{LSND}}^{2}}$, we have

$$
\left.|| U_{e 3}\right|^{2}-\left|U_{e 4}\right|^{2}\left|\sqrt{\Delta m_{\mathrm{LSND}}^{2}} \lesssim\right|\langle m\rangle \mid \lesssim\left(\left|U_{e 3}\right|^{2}+\left|U_{e 4}\right|^{2}\right) \sqrt{\Delta m_{\mathrm{LSND}}^{2}}
$$

Since the quantity $\left|U_{e 3}\right|^{2}+\left|U_{e 4}\right|^{2}$ is large in scheme A (see Eq. (3.10)), we obtain

$$
\left.|| U_{e 3}\right|^{2}-\left|U_{e 4}\right|^{2}\left|\sqrt{\Delta m_{\mathrm{LSND}}^{2}} \lesssim\right|\langle m\rangle \mid \lesssim \sqrt{\Delta m_{\mathrm{LSND}}^{2}} .
$$

Furthermore, from the inequality (3.10) one can see that the contribution of the mixing of $\nu_{e}$ with $\nu_{1}$ and $\nu_{2}$ to the survival probability of solar electron neutrinos is negligible and $\left|U_{e 3}\right|^{2}$ and $\left|U_{e 4}\right|^{2}$ are related to the mixing angle $\vartheta_{\text {sun }}$ obtained from the two-generation fit of solar neutrino experiments by

$$
\left|U_{e 3}\right|^{2} \simeq \cos ^{2} \vartheta_{\text {sun }}, \quad\left|U_{e 4}\right|^{2} \simeq \sin ^{2} \vartheta_{\text {sun }} .
$$

Hence, the range (3.15) can be written as

$$
\sqrt{\left(1-\sin ^{2} 2 \vartheta_{\text {sun }}\right) \Delta m_{\mathrm{LSND}}^{2}} \lesssim|\langle m\rangle| \lesssim \sqrt{\Delta m_{\mathrm{LSND}}^{2}}
$$

Let us emphasize that this allowed range for $|\langle m\rangle|$ in scheme A depends only on the assumption that massive neutrinos are Majorana particles.

In the case of the SMA-MSW solution of the solar neutrino problem (for both $\nu_{e} \rightarrow \nu_{\tau}$ or $\nu_{e} \rightarrow \nu_{s}$ transitions) $\sin ^{2} 2 \vartheta_{\text {sun }}$ is very small $\left(\sin ^{2} 2 \vartheta_{\text {sun }} \lesssim 10^{-2}\right)$ and we have

$$
|\langle m\rangle| \simeq \sqrt{\Delta m_{\mathrm{LSND}}^{2}} \simeq 0.45-1.4 \mathrm{eV} \quad(\mathrm{SMA}-\mathrm{MSW}) .
$$

Hence, the experimental upper bound (1.3) indicates that the SMA-MSW solution of the solar neutrino problem is disfavored in scheme $A$.

Furthermore, the upper bound $N_{\nu}^{\mathrm{BBN}}<4$ for the effective number of neutrinos in BigBang Nucleosynthesis (BBN) (see, for example, [60]) implies that [54,57,58]

$$
\left|U_{s 1}\right|^{2}+\left|U_{s 2}\right|^{2} \lesssim 10^{-2}
$$

in scheme A. The analysis of recent astrophysical data yields the upper bound $N_{\nu}^{\mathrm{BBN}} \leq 3.2$ at 95\% CL [61], although the issue is still rather controversial (see [62,63]). The inequalities (3.10) and (3.19), together with the unitarity of the mixing matrix, imply that the oscillations of solar neutrinos occur mainly in the $\nu_{e} \rightarrow \nu_{s}$ channel [57,58]. In this case, the analysis of solar neutrino data in terms of two-generation $\nu_{e} \rightarrow \nu_{s}$ oscillations is valid in the four-neutrino scheme $\mathrm{A}$ if the usual two-generation mixing parameters $\Delta m^{2}$ and $\vartheta$ are identified, respectively, with $\Delta m_{\text {sun }}^{2}=\Delta m_{43}^{2}$ and $\vartheta_{\text {sun }}$ defined in Eq. (3.16) (from Eqs. (3.10), (3.16), (3.19) and the unitarity of the mixing matrix we obtain $\left|U_{s 3}\right|^{2} \simeq \sin ^{2} \vartheta_{\text {sun }}$

${ }^{3}$ The bound $N_{\nu}^{\mathrm{BBN}} \leq 3.2$ 61 implies that $\left|U_{s 1}\right|^{2}+\left|U_{s 2}\right|^{2} \lesssim 5 \times 10^{-4}$ 57,58 in scheme A! 
and $\left.\left|U_{s 4}\right|^{2} \simeq \cos ^{2} \vartheta_{\text {sun }}\right)$. The results of the analyses of solar neutrino data in terms of twogeneration $\nu_{e} \rightarrow \nu_{s}$ oscillations show that only the SMA-MSW solution is allowed [38, 42, 43. Therefore, comparing Eqs. (1.3) and (3.18) we conclude that the scheme $A$ is disfavored by the experimental upper bound $|\langle m\rangle|$ and the $B B N$ bound $N_{\nu}^{\mathrm{BBN}}<4$.

Summarizing, the data from oscillation experiments, from neutrinoless double- $\beta$ decay experiments and from the measurement of the abundances of primordial elements indicate that, among all the possible four-neutrino schemes there is only one allowed, scheme B.

\section{B. Scheme B}

In scheme B, the BBN upper bound $N_{\nu}^{\mathrm{BBN}}<4$ implies that 57,58

$$
\left|U_{s 3}\right|^{2}+\left|U_{s 4}\right|^{2} \lesssim 10^{-4}
$$

From this inequality, Eq. (3.11) and the unitarity of the mixing matrix it follows that the oscillations of solar neutrinos occur mainly in the $\nu_{e} \rightarrow \nu_{s}$ channel. Therefore, the analysis of solar neutrino data in terms of two-generation $\nu_{e} \rightarrow \nu_{s}$ oscillations is valid in the fourneutrino scheme B if the usual two-generation mixing parameters $\Delta m^{2}$ and $\vartheta$ are identified, respectively, with $\Delta m_{\text {sun }}^{2}=\Delta m_{21}^{2}$ and $\vartheta_{\text {sun }}$ defined by

$$
\left|U_{e 1}\right|^{2} \simeq \cos ^{2} \vartheta_{\text {sun }}, \quad\left|U_{e 2}\right|^{2} \simeq \sin ^{2} \vartheta_{\text {sun }}
$$

(from Eqs. (3.11), (3.20), (3.21) and the unitarity of the mixing matrix we obtain $\left|U_{s 1}\right|^{2} \simeq$ $\sin ^{2} \vartheta_{\text {sun }}$ and $\left.\left|U_{s 2}\right|^{2} \simeq \cos ^{2} \vartheta_{\text {sun }}\right)$. Since the results of the analyses of solar neutrino data in terms of two-generation $\nu_{e} \rightarrow \nu_{s}$ oscillations [38,42, 43] show that only the SMA-MSW solution is allowed, with $10^{-3} \lesssim \sin ^{2} 2 \vartheta_{\text {sun }} \lesssim 10^{-2}$, we have $2.5 \times 10^{-4} \lesssim\left|U_{e 2}\right|^{2} \lesssim 2.5 \times 10^{-3}$. Therefore, in scheme B we have

$$
\left|U_{e 1}\right|^{2} \simeq 1, \quad\left|U_{e 2}\right|^{2},\left|U_{e 3}\right|^{2},\left|U_{e 4}\right|^{2} \ll 1 .
$$

In the scheme B there are two possibilities: a quasi-degenerate mass spectrum

$$
\underbrace{\overbrace{m_{1}<m_{2}}^{\text {sun }} \lesssim \overbrace{m_{3}<m_{4}}^{\text {atm }}}_{\text {LSND }}
$$

or a mass hierarchy

$$
\underbrace{\overbrace{m_{1}<m_{2}}^{\text {sun }} \ll \overbrace{m_{3}<m_{4}}^{\text {atm }}}_{\text {LSND }}
$$

If the quasi-degenerate mass spectrum BD is realized in nature, it is clear that from Eqs. (1.1) and (3.22) we have

$$
|\langle m\rangle| \simeq m_{1}
$$

In this case, the experimental upper bound (1.3) implies that 


$$
m_{1} \lesssim 0.2-0.4 \mathrm{eV}
$$

The observation of neutrinoless double- $\beta$ decay by the next generation of experiments, which are sensitive to values of $|\langle m\rangle|$ in the range $10^{-2}-10^{-1} \mathrm{eV}$ [17], together with the confirmation of the four-neutrino scheme $\mathrm{B}$ by neutrino oscillation experiments, will provide an evidence in favor of the quasi-degenerate scheme BD.

If the hierarchical mass spectrum $\mathrm{BH}$ is realized in nature, the absence of unlikely finetuned cancellations between the contributions of $m_{1}, m_{2}$ and $m_{3}, m_{4}$ to the effective Majorana mass (1.1) implies that

$$
|\langle m\rangle| \simeq \max \left[|\langle m\rangle|_{12},|\langle m\rangle|_{34}\right]
$$

with

$$
\begin{aligned}
& |\langle m\rangle|_{12} \equiv\left|U_{e 1}^{2} m_{1}+U_{e 2}^{2} m_{2}\right| \\
& |\langle m\rangle|_{34} \equiv\left|U_{e 3}^{2} m_{3}+U_{e 4}^{2} m_{4}\right|
\end{aligned}
$$

From Eq. (3.22), if $m_{1} \simeq m_{2}$ we have $|\langle m\rangle|_{12} \simeq m_{2}$ and the contribution of $|\langle m\rangle|_{12}$ to $|\langle m\rangle|$ could be sizable. On the other hand, if $m_{1} \ll m_{2}$ we have $|\langle m\rangle|_{12} \ll m_{2}$ and the contribution of $|\langle m\rangle|_{12}$ to $|\langle m\rangle|$ is strongly suppressed. In this case, if there are no unlikely fine-tuned cancellations between the contributions of $m_{1}$ and $m_{2}$ to $|\langle m\rangle|_{12}$, we have $|\langle m\rangle|_{12} \simeq|\langle m\rangle|_{2}$ with $|\langle m\rangle|_{2}$ in the range (2.11). In any case, at present it is not known if $m_{1} \simeq m_{2}$ or $m_{1} \ll m_{2}$ and we cannot infer the contribution of $|\langle m\rangle|_{12}$ to $|\langle m\rangle|$.

Let us consider now the contribution $|\langle m\rangle|_{34}$ to the effective Majorana mass. In principle it is possible that $|\langle m\rangle|_{34}=0$ if $U_{e 3}^{2} m_{3}+U_{e 4}^{2} m_{4}=0$, i.e.

$$
\left|U_{e 3}\right|^{2} m_{3}=\left|U_{e 4}\right|^{2} m_{4}
$$

and

$$
\left|\arg \left(U_{e 3}\right)-\arg \left(U_{e 4}\right)\right|=\pi / 2
$$

(the condition (3.31) is satisfied if $\mathrm{CP}$ is conserved and $\nu_{3}$ and $\nu_{4}$ have opposite $\mathrm{CP}$ parities [64, 24, 22,27]). However, even if $m_{3} \simeq m_{4}$, since $\left|U_{e 3}\right|^{2}+\left|U_{e 4}\right|^{2} \ll 1$ (see Eq. (3.11)), there is no reason to have $\left|U_{e 3}\right|^{2} \simeq\left|U_{e 4}\right|^{2}$. On the other hand, the explanation of the atmospheric neutrino data with $\nu_{\mu} \rightarrow \nu_{\tau}$ oscillations [9 13], that is favored by the latest Super-Kamiokande data [65], requires a large mixing in the $\nu_{\mu}, \nu_{\tau}-\nu_{3}, \nu_{4}$ sector which could be related to the fact that $\left|U_{\mu 3}\right|^{2}+\left|U_{\mu 4}\right|^{2}$ and $\left|U_{\tau 3}\right|^{2}+\left|U_{\tau 4}\right|^{2}$ are large (close to one) and $m_{3} \simeq m_{4}$.

Therefore, in the following we will assume that $\left|U_{e 3}\right|^{2}$ and $\left|U_{e 4}\right|^{2}$ have different orders of magnitude. In this case, the contribution of $m_{3}$ and $m_{4}$ to the effective Majorana mass is given by

$$
|\langle m\rangle|_{34} \simeq d_{e} \sqrt{\Delta m_{\mathrm{LSND}}^{2}}
$$

where we have taken into account that $m_{3} \simeq m_{4} \simeq \sqrt{\Delta m_{41}^{2}}=\sqrt{\Delta m_{\mathrm{LSND}}^{2}}$ and we have defined 


$$
d_{\alpha} \equiv \sum_{k=3,4}\left|U_{\alpha k}\right|^{2} \quad(\alpha=e, \mu, \tau, s)
$$

It has been shown in 55 that $d_{e}$ is small in scheme B:

$$
d_{e} \leq a_{e}^{\text {Bugey }}
$$

with

$$
a_{e}^{\text {Bugey }}=\frac{1}{2}\left(1-\sqrt{1-\sin ^{2} 2 \vartheta_{\text {Bugey }}}\right) .
$$

Here $\sin ^{2} 2 \vartheta_{\text {Bugey }}$ is the upper value of the two-neutrino mixing parameter $\sin ^{2} 2 \vartheta$ obtained from the Bugey exclusion curve [51] as a function of $\Delta m^{2}=\Delta m_{\mathrm{LSND}}^{2}$, where $\Delta m^{2}$ is the twoneutrino mass-squared difference used in the analysis of the Bugey data (the upper bound (3.11) has been obtained from the inequality (3.34) restricting $\Delta m_{\text {LSND }}^{2}$ in the LSND-allowed range $(3.4))$.

From Eqs. (3.32) and (3.34), for $|\langle m\rangle|_{34}$ we obtain the upper bound 25 27]

$$
|\langle m\rangle|_{34} \lesssim a_{e}^{\text {Bugey }} \sqrt{\Delta m_{\mathrm{LSND}}^{2}}
$$

The numerical value of this upper bound as a function of $\Delta m_{\mathrm{LSND}}^{2}$ is depicted by the solid line in Fig. 2. The dashed line in Fig. 2 represents the unitarity limit $|\langle m\rangle|_{34} \leq \sqrt{\Delta m_{\mathrm{LSND}}^{2}}$.

The amplitude $A_{\mu e}=4\left|\sum_{k=3,4} U_{e k} U_{\mu k}^{*}\right|^{2}$ of short-baseline $\bar{\nu}_{\mu} \rightarrow \bar{\nu}_{e}$ oscillations in scheme $\mathrm{B}$ is bounded by 55

$$
A_{\mu e} \leq 4 d_{e} d_{\mu}
$$

Since $d_{\mu}$ is large in scheme B [55], we have

$$
d_{e} \geq \frac{A_{\mu e}^{\min }}{4}
$$

where $A_{\mu e}^{\mathrm{min}}$ is the minimum value of $A_{\mu e}$ measured in the LSND experiment. The physical reason of this lower bound for $d_{e}$ is that $\nu_{e}$ must have some mixing with $\nu_{3}$ and/or $\nu_{4}$ in order to generate the oscillations observed in the LSND experiment.

Taking into account Eq. (3.32), the inequality (3.38) leads to the lower bound

$$
|\langle m\rangle|_{34} \gtrsim \frac{A_{\mu e}^{\min }}{4} \sqrt{\Delta m_{\mathrm{LSND}}^{2}} .
$$

The numerical value of this lower bound as a function of $\Delta m_{\text {LSND }}^{2}$ is shown in Fig. 2 by the dotted curve that, together with the solid line obtained from the upper bound (3.36) defines an allowed region in the $\Delta m_{\mathrm{LSND}}^{2}-|\langle m\rangle|_{34}$ plane (shadowed area). From Fig. 2 one can see that

$$
6.9 \times 10^{-4} \mathrm{eV} \lesssim|\langle m\rangle|_{34} \lesssim 2.1 \times 10^{-2} \mathrm{eV}
$$


Summarizing, in the framework of the scheme BH in Eq. (3.24) we have made three assumptions: (i) massive neutrinos are Majorana particles, (ii) there is no unlikely fine-tuned cancellations between the contributions of $m_{1}, m_{2}$ and $m_{3}, m_{4}$ to the effective Majorana mass $|\langle m\rangle|$, (iii) the two small elements $U_{e 3}$ and $U_{e 4}$ of the neutrino mixing matrix have different orders of magnitude. Under these reasonable assumptions, we have obtained the following allowed range for the effective Majorana mass in $\beta \beta_{0 \nu}$ decay:

$$
7 \times 10^{-4} \mathrm{eV} \lesssim|\langle m\rangle| \lesssim 2 \times 10^{-2} \mathrm{eV},
$$

from the contribution of $m_{3}$ and $m_{4}$ alone (the effective Majorana mass could be even larger than $2 \times 10^{-2} \mathrm{eV}$ if $m_{1} \simeq m_{2} \gtrsim 2 \times 10^{-2} \mathrm{eV}$ ). Such values of the effective Majorana mass could be measured by future $\beta \beta_{0 \nu}$ decay experiments [17, [1]].

\section{CONCLUSIONS}

We have derived lower limits for the the effective Majorana mass in neutrinoless double- $\beta$ decay in the scheme with mixing of three neutrinos and a mass hierarchy [Eq. (2.2)] under the natural assumptions that massive neutrinos are Majorana particles and there are no large cancellations among the contributions of the different neutrino masses. If there is a hierarchy of neutrino masses, large cancellations are unlikely (unless an unknown symmetry is at work), because they require a fine-tuning among the values of the neutrino masses and the elements of the neutrino mixing matrix, which are independent quantities.

Under the only assumption that massive neutrinos are Majorana particles, we have shown that, among all the possible four-neutrino schemes that can accommodate the results of solar and atmospheric experiments and the results of the LSND experiment, only the scheme B [Eq. (3.9)] is compatible with the experimental results on neutrinoless double- $\beta$ decay and the measurements of the cosmic abundances of elements produced in Big-Bang Nucleosynthesis. In the scheme $\mathrm{B}$ there are two possibilities: the quasi-degenerate mass spectrum $\mathrm{BD}$ [Eq. (3.23)] and the hierarchical mass spectrum BH [Eq. (3.24)].

If the quasi-degenerate four-neutrino scheme $\mathrm{BD}$ is realized in nature, neutrinoless double- $\beta$ decay should be observed by the next generation of experiments, which will be sensitive to $|\langle m\rangle| \sim 10^{-2}-10^{-1} \mathrm{eV}$.

In the framework of the hierarchical four-neutrino scheme $\mathrm{BH}$, we have shown that there is a lower bound for the effective Majorana mass in $\beta \beta_{0 \nu}$ decay, under the assumptions that massive neutrinos are Majorana particles, there are no large cancellations among the contributions of $m_{1}, m_{2}$ and $m_{3}, m_{4}$ and the two small elements $U_{e 3}$ and $U_{e 4}$ of the neutrino mixing matrix have different orders of magnitude.

We hope that the indications presented here in favor of a lower bound, albeit small, for the effective Majorana mass in neutrinoless double- $\beta$ decay will encourage the development

of future $\beta \beta_{0 \nu}$ experiments. 


\section{REFERENCES}

[1] S.M. Bilenky and B. Pontecorvo, Phys. Rep. 41, 225 (1978); S.M. Bilenky and S.T. Petcov, Rev. Mod. Phys. 59, 671 (1987); C.W. Kim and A. Pevsner, Neutrinos in Physics and Astrophysics, Contemporary Concepts in Physics, Vol. 8, Harwood Academic Press, Chur, Switzerland, 1993.

[2] S.M. Bilenky, C. Giunti and W. Grimus, hep-ph/9812360 [to be published in Prog. Part. Nucl. Phys., Vol. 43].

[3] P. Fisher, B. Kayser, K.S. McFarland, hep-ph/9906244.

[4] B.T. Cleveland et al., Astrophys. J. 496, 505 (1998).

[5] K.S. Hirata et al., Phys. Rev. Lett. 77, 1683 (1996).

[6] W. Hampel et al., Phys. Lett. B388, 384 (1996); Phys. Lett. B447, 127 (1999).

[7] D.N. Abdurashitov et al., Phys. Rev. Lett. 77, 4708 (1996); Phys. Rev. C59, 2246 (1999).

[8] Y. Fukuda, Phys. Rev. Lett. 81, 1158 (1998), Erratum ibid. 81, 4279 (1998), hepex/9805021; Phys. Rev. Lett. 82, 2430 (1999), hep-ex/9812011; M.B. Smy (SuperKamiokande Coll.), hep-ex/9903034.

[9] Y. Fukuda et al., Phys. Lett. B335, 237 (1994).

[10] R. Becker-Szendy et al., Nucl. Phys. B (Proc. Suppl.) 38, 331 (1995).

[11] Y. Fukuda et al., Phys. Rev. Lett. 81, 1562 (1998), hep-ex/9807003; Phys. Rev. Lett. 82, 2644 (1999), hep-ex/9812014; A. Habig (Super-Kamiokande Coll.), hep-ex/9903047; K. Scholberg (Super-Kamiokande Coll.), hep-ex/9905016.

[12] W.W.M. Allison et al., Phys. Lett. B449, 137 (1999).

[13] M. Ambrosio et al., Phys. Lett. B434, 451 (1998); F. Ronga (MACRO Coll.), hepex/9905025; A. Surdo (MACRO Coll.), hep-ex/9905028.

[14] C. Athanassopoulos et al., Phys. Rev. Lett. 75, 2650 (1995); Phys. Rev. Lett. 77, 3082 (1996); Phys. Rev. Lett. 81, 1774 (1998).

[15] G. Mills (LSND Coll.), Talk presented at the XXXIV ${ }^{\text {th }}$ Rencontres de Moriond Electroweak Interactions and Unified Theories, Les Arcs, 13-20 March 1999 (scanned transparencies available at http://moriond.in2p3.fr/EW/transparencies).

[16] M. Moe and P. Vogel, Annu. Rev. Nucl. Part. Sci. 44, 247 (1994); A. Morales, Nucl. Phys. B (Proc. Suppl.) 77, 335 (1999), hep-ph/9809540.

[17] C.E. Aalseth et al., Nucl. Phys. B (Proc. Suppl.) 70, 236 (1999); X. Sarazin (NEMO Coll.), ibid. 70, 239 (1999); V.D. Ashitkov et al., ibid. 70, 233 (1999); E. Fiorini, Phys. Rep. 307, 309 (1998).

[18] L. Baudis et al., Phys. Rev. Lett. 83, 41 (1999), hep-ex/9902014.

[19] J. Hellmig and H.V. Klapdor-Kleingrothaus, Z. Phys. A359, 351 (1997); H.V. KlapdorKleingrothaus and M. Hirsch, Z. Phys. A359, 361 (1997); H.V. Klapdor-Kleingrothaus, J. Hellmig and M. Hirsch, J. Phys. G24, 483 (1998).

[20] A.I. Belesev, Phys. Lett. B350, 263 (1995); H. Barth et al., Prog. Part. Nucl. Phys. 40, 353 (1998).

[21] C. Caso et al., Eur. Phys. J. C3, 1 (1998).

[22] S.T. Petcov and A.Yu. Smirnov, Phys. Lett. B322, 109 (1994).

[23] S.M. Bilenky, A. Bottino, C. Giunti and C.W. Kim, Phys. Lett. B356, 273 (1995), hep-ph/9504405; Phys. Rev. D54, 1881 (1996), hep-ph/9602216. 
[24] S.M. Bilenky, C. Giunti, C.W. Kim and S.T. Petcov, Phys. Rev. D54, 4432 (1996).

[25] S.M. Bilenky, C. Giunti, C.W. Kim and M. Monteno, Phys. Rev. D57, 6981 (1998), hep-ph/9711400.

[26] S.M. Bilenky, C. Giunti and W. Grimus, Nucl. Phys. B (Proc. Suppl.) 77, 151 (1999), hep-ph/9809368; S.M. Bilenky and C. Giunti, Talk presented at WIN99, Cape Town, South Africa, 24-30 January 1999, hep-ph/9904328.

[27] S.M. Bilenky, C. Giunti, W. Grimus, B. Kayser and S.T. Petcov, hep-ph/9907234] [to be published in Phys. Lett. B].

[28] F. Vissani, hep-ph/9708483; hep-ph/9904349; JHEP 9906022 (1999), hep-ph/9906525.

[29] H. Minakata and O. Yasuda, Phys. Rev. D56, 1692 (1997); Nucl. Phys. B523, 597 (1998).

[30] T. Fukuyama, K. Matsuda and H. Nishiura, Phys. Rev. D57, 5844 (1998); hep$\mathrm{ph} / 9804262$.

[31] R. Adhikari and G. Rajasekaran, hep-ph/9812361.

[32] G.C. Branco, M.N. Rebelo and J.I. Silva-Marcos, Phys. Rev. Lett. 82, 683 (1999).

[33] V. Barger and K. Whisnant, Phys. Lett. B 456, 194 (1999), hep-ph/9904281.

[34] A. Staudt, K. Muto and H.V. Klapdor-Kleingrothaus, Europhys. Lett. 13, 31 (1990); T. Tomoda, Rep. Prog. Phys. 54, 53 (1991); W. Haxton et al., Nucl. Phys. B (Proc. Suppl.) 31, 82 (1993); X.R. Wu et al., Phys. Lett. B272, 169 (1991); Phys. Lett. B276, 274 (1992); F. Simkovic et al., Phys. Lett. B393, 267 (1997).

[35] F. Simkovic et al., hep-ph/9905509.

[36] E. Caurier et al., Phys. Rev. Lett. 77, 1954 (1996).

[37] P. Lipari, hep-ph/9904443.

[38] J.N. Bahcall, P.I. Krastev and A.Yu. Smirnov, Phys. Rev. D58, 096016 (1998), hepph/9807216.

[39] V. Berezinsky, hep-ph/9904259.

[40] K. Eitel (KARMEN Coll.), Proc. to Lake Louise Winter Institute 1999, 14-20 Feb. 1999, Lake Louise; T. Jannakos (KARMEN Coll.), Proc. to Les Rencontres De Moriond 1999, 13-20 March 1999, Les Arc 1800; KARMEN WWW page: http://www-ik1.fzk.de/www/karmen/karmen_e.html.

[41] Booster Neutrino Experiment (BooNE), http://nu1.lampf.lanl.gov/BooNE; I-216 $\nu_{\mu} \rightarrow$ $\nu_{e}$ proposal at CERN, http://chorus01.cern.ch/pucchel/loi/; Oak Ridge Large Neutrino Detector (ORLaND), http://www.phys.subr.edu/orland/; Neutrinos at the European Spallation Source (NESS), http://www.isis.rl.ac.uk/ess/neut\%5Fess.htm.

[42] Y. Fukuda et al., Phys. Rev. Lett. 82, 1810 (1999), hep-ex/9812009.

[43] M.C. Gonzalez-Garcia et al., hep-ph/9906469.

[44] M.C. Gonzalez-Garcia et al., Phys. Rev. D58, 033004 (1998), hep-ph/9801368; Nucl. Phys. B543, 3 (1999), hep-ph/9807305.

[45] G.L. Fogli, E. Lisi, A. Marrone and G. Scioscia, Phys. Rev. D59, 033001 (1999), hepph/9808205.

[46] M. Gell-Mann, P. Ramond and R. Slansky, in Supergravity, p. 315, edited by F. van Nieuwenhuizen and D. Freedman, North Holland, Amsterdam, 1979; T. Yanagida, Proc. of the Workshop on Unified Theory and the Baryon Number of the Universe, KEK, Japan, 1979; R.N. Mohapatra and G. Senjanović, Phys. Rev. Lett. 44, 912 (1980).

[47] M. Apollonio et al., Phys. Lett. B420, 397 (1998). 
[48] S.M. Bilenky and C. Giunti, Phys. Lett. B444, 379 (1998), hep-ph/9802201.

[49] S.P. Mikheyev and A.Yu. Smirnov, Yad. Fiz. 42, 1441 (1985) [Sov. J. Nucl. Phys. 42, 913 (1985)]; Il Nuovo Cimento C9, 17 (1986); L. Wolfenstein, Phys. Rev. D17, 2369 (1978); Phys. Rev. D20, 2634 (1979).

[50] J.N. Bahcall, P.I. Krastev and A.Yu. Smirnov, hep-ph/9905220.

[51] B. Achkar et al., Nucl. Phys. B434, 503 (1995).

[52] J.T. Peltoniemi, D. Tommasini and J.W.F. Valle, Phys. Lett. B298, 383 (1993); E.J. Chun et al., Phys. Lett. B357, 608 (1995); S.C. Gibbons et al., Phys. Lett. B430, 296 (1998); B. Brahmachari and R.N. Mohapatra, Phys. Lett. B437, 100 (1998); S. Mohanty, D.P. Roy and U. Sarkar, Phys. Lett. B445, 185 (1998); J.T. Peltoniemi and J.W.F. Valle, Nucl. Phys. B406, 409 (1993); Q.Y. Liu and A.Yu. Smirnov, Nucl. Phys. B524, 505 (1998); D.O. Caldwell and R.N. Mohapatra, Phys. Rev. D48, 3259 (1993); E. Ma and P. Roy, Phys. Rev. D52, R4780 (1995); A.Yu. Smirnov and M. Tanimoto, Phys. Rev. D55, 1665 (1997); N. Gaur et al., Phys. Rev. D58, 071301 (1998); E.J. Chun, C.W. Kim and U.W. Lee, Phys. Rev. D58, 093003 (1998); K. Benakli and A.Yu. Smirnov, Phys. Rev. Lett. 79, 4314 (1997); Y. Chikira, N. Haba and Y. Mimura, hep-ph/9808254; C. Liu and J. Song, hep-ph/9812381; W. Grimus, R. Pfeiffer and T. Schwetz, hep-ph/9905320.

[53] J.J. Gomez-Cadenas and M.C. Gonzalez-Garcia, Z. Phys. C71, 443 (1996), hepph/9504246; S. Goswami, Phys. Rev. D55, 2931 (1997), hep-ph/9507212; S.M. Bilenky, C. Giunti, C.W. Kim and S.T. Petcov, Phys. Rev. D54, 4432 (1996), hep-ph/9604364; V. Barger, Y.B. Dai, K. Whisnant and B.L. Young, Phys. Rev. D59, 113010 (1999), hep-ph/9901388; V. Barger, T.J. Weiler and K. Whisnant, Phys. Lett. B427, 97 (1998), hep-ph/9712495.

[54] N. Okada and O. Yasuda, Int. J. Mod. Phys. A12, 3669 (1997), hep-ph/9606411.

[55] S.M. Bilenky, C. Giunti and W. Grimus, Proc. of Neutrino '96, Helsinki, June 1996, edited by K. Enqvist et al., p. 174, World Scientific, 1997 (hep-ph/9609343); Eur. Phys. J. C1, 247 (1998), hep-ph/9607372; S.M. Bilenky, C. Giunti, W. Grimus and T. Schwetz, hep-ph/9903454.

[56] S.M. Bilenky, C. Giunti and W. Grimus, Phys. Rev. D57, 1920 (1998), hep-ph/9710209; Phys. Rev. D58, 033001 (1998), hep-ph/9712537.

[57] S.M. Bilenky, C. Giunti, W. Grimus and T. Schwetz, hep-ph/9804421] [to be published in Astropart. Phys.].

[58] S.M. Bilenky, C. Giunti, W. Grimus and T. Schwetz, hep-ph/9807569, Talk presented at the Ringberg Euroconference "New Trends in Neutrino Physics", 24-29 May 1998; in New Era in Neutrino Physics, p. 179, edited by H. Minakata and O. Yasuda, Universal Academy Press, 1999, hep-ph/9809466.

[59] A. Yu. Smirnov, hep-ph/9901208; R.N. Mohapatra, hep-ph/9903261.

[60] D.N. Schramm and M.S. Turner, Rev. Mod. Phys. 70, 303 (1998), astro-ph/9706069.

[61] S. Burles, K.M. Nollett, J.N. Truran and M.S. Turner, Phys. Rev. Lett. 82, 4176 (1999), astro-ph/9901157.

[62] K.A. Olive, astro-ph/9903309.

[63] E. Lisi, S. Sarkar and F.L. Villante, Phys. Rev. D59, 123520 (1999), hep-ph/9901404; S. Sarkar, astro-ph/9903183.

[64] S.M. Bilenky, N.P. Nedelcheva and S.T. Petcov, Nucl. Phys. B247, 61 (1984); B. Kayser, 
Phys. Rev. D30, 1023 (1984).

[65] J. Learned, Talk presented at the $23^{\text {rd }}$ Johns Hopkins Workshop on Current Problems in Particle Theory Neutrinos in the Next Millennium, Baltimore, 10-12 June 1999. 


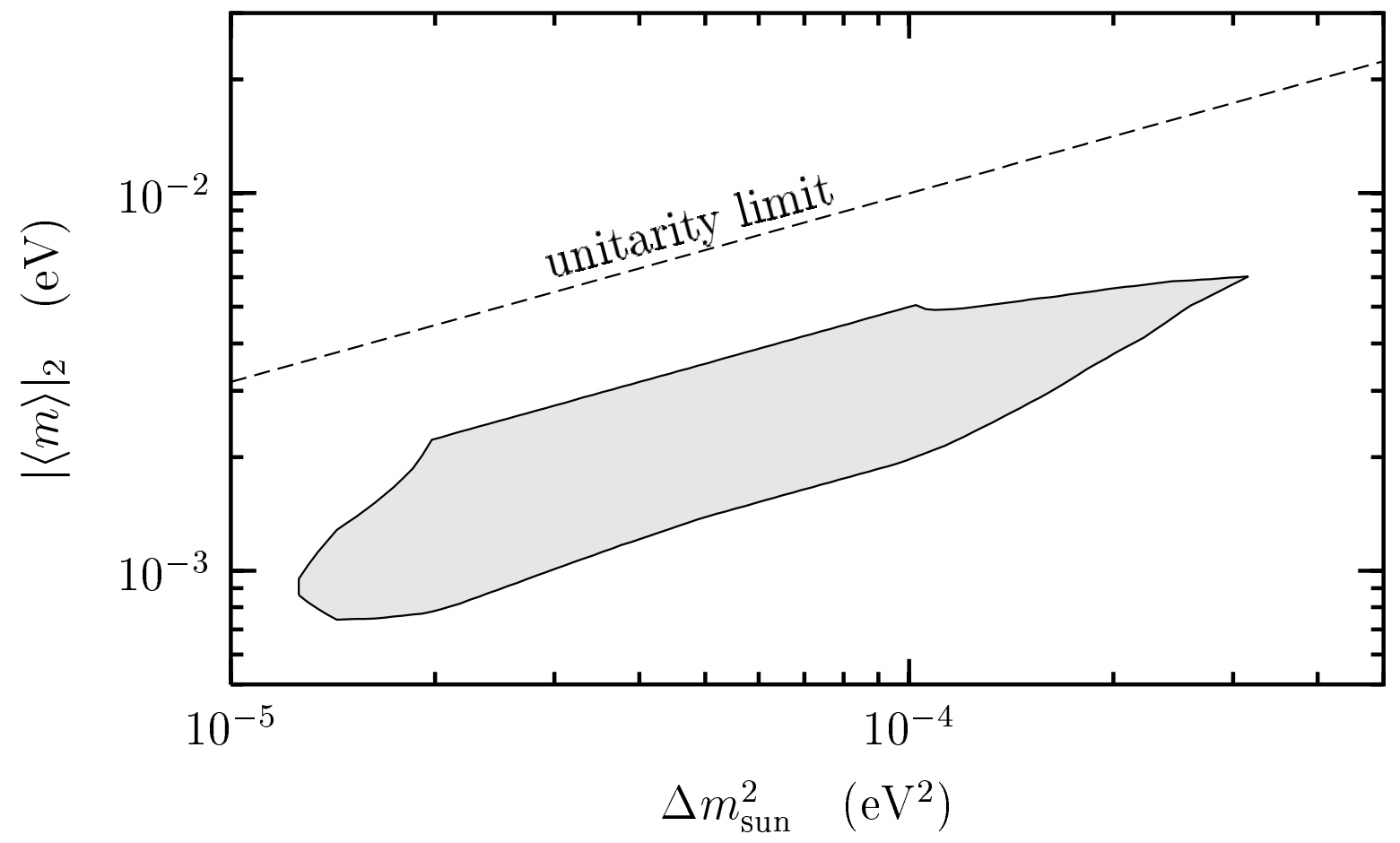

FIG. 1. The shadowed area shows the allowed range for $|\langle m\rangle|_{2}$ as a function of $\Delta m_{\text {sun }}^{2}$ in the scheme with mixing of three neutrinos with a mass hierarchy discussed in Section II, in the case of the LMA-MSW solution of the solar neutrino problem. The shadowed area has been obtained using Eq. (2.6) and the allowed range for $\sin ^{2} 2 \vartheta_{\text {sun }}$ given by the LMA-MSW region (99\% CL) in the $\sin ^{2} 2 \vartheta_{\text {sun }}-\Delta m_{\text {sun }}^{2}$ plane presented in Fig. 2 of Ref. [42]. The dashed line represents the unitarity limit $|\langle m\rangle|_{2} \leq \sqrt{\Delta m_{\text {sun }}^{2}}$ 


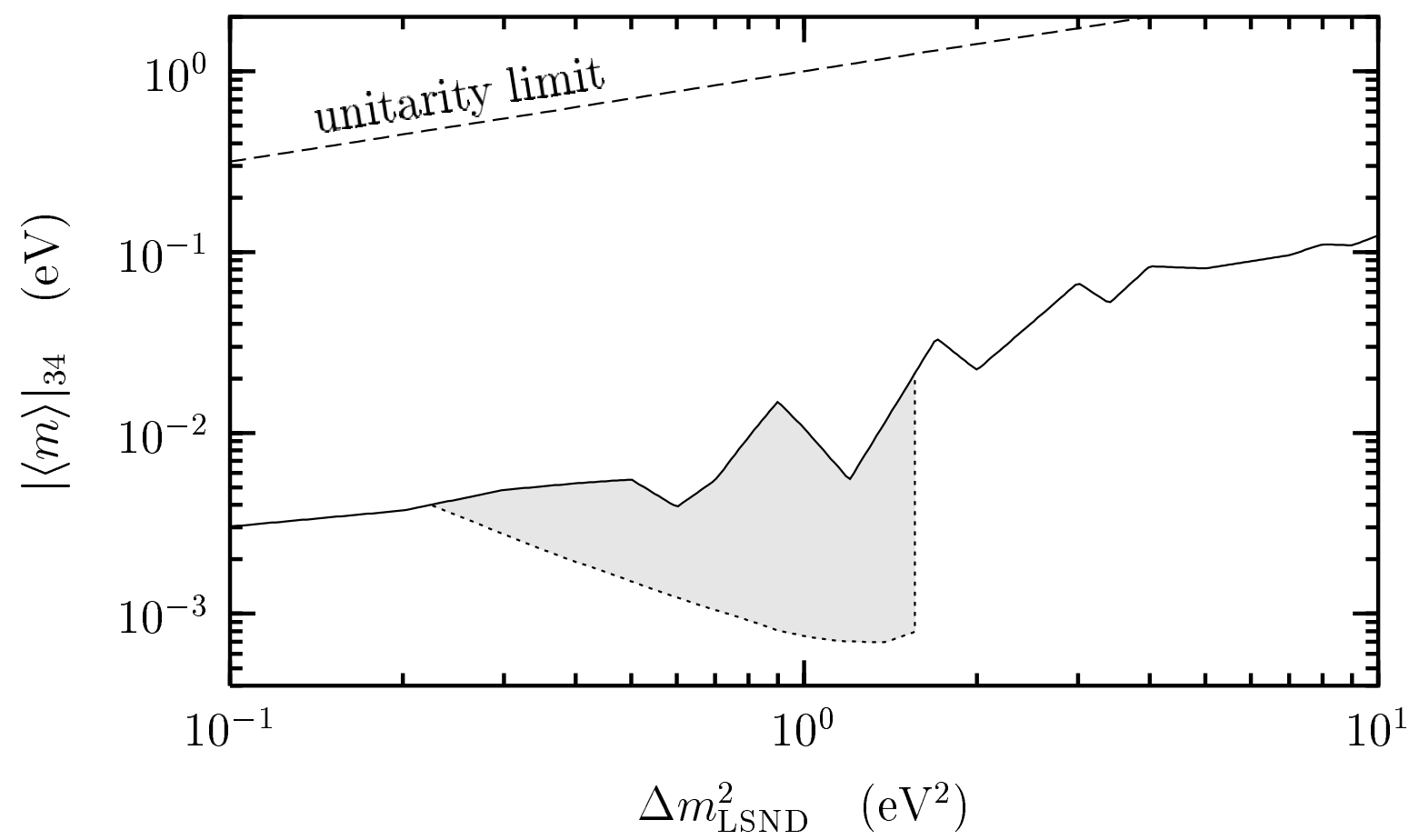

FIG. 2. The shadowed region shows the allowed range for $|\langle m\rangle|_{34}$ as a function of $\Delta m_{\mathrm{LSND}}^{2}$ in the four-neutrino scheme BH (see Eq. (3.24)). The solid line represents the upper bound in Eq. (3.36) and the dotted line represents the lower bound in Eq. (3.39). The dashed line represents the unitarity limit $|\langle m\rangle|_{34} \leq \sqrt{\Delta m_{\mathrm{LSND}}^{2}}$. 